\title{
A simplified method for parameters calibration of the new local approach model for cleavage fracture in a ferritic steel
}

\author{
Airu Shen ${ }^{\mathrm{a}}$, Peichao $\mathrm{Li}^{\mathrm{a}, *}$, Keyong Wang ${ }^{\mathrm{a}}$, Guian Qian ${ }^{\mathrm{b}, *}$, Filippo Berto ${ }^{\mathrm{c}}$ \\ ${ }^{a}$ School of Mechanical and Automotive Engineering, Shanghai University of Engineering Science, Shanghai 201620, China \\ ${ }^{\mathrm{b}}$ State Key Laboratory of Nonlinear Mechanics (LNM), Institute of Mechanics, Chinese Academy of Sciences, Beijing 100190, China \\ ${ }^{c}$ Department of Mechanical and Industrial Engineering, Norwegian University of Science and Technology (NTNU), Richard Birkelands vei 2b, 7491 Trondheim, Norway
}

\section{A R T I C L E I N F O}

\section{Keywords:}

Parameters calibration

NLAM

Cleavage fracture

Weibull parameters

Threshold coefficient of fracture process zone

\begin{abstract}
A B S T R A C T
This work presents a simplified method for parameters calibration of the new local approach model (NLAM) for cleavage fracture in a ferritic steel. Based on the Beremin model, a NLAM was recently proposed by Lei (2016) to calculate the Weibull parameters. Although the above NLAM proves to be mathematically and physically selfconsistent, yet there are still no reliable and convenient methods to calibrate the model parameters. Considering the plastic volume is determined by the threshold coefficient of fracture process zone $\lambda$, we adopt four different values of $\lambda$ in this work to calculate the plastic volume so as to obtain four groups of calibrated Weibull parameters. The material used in this study is the pressure vessel steel 20MnMoNi55 with some published strength data under two subzero temperatures. And the finite element analysis FEA) software ABAQUS is employed to obtain the stress distributions, then the calibration results for four different values of $\lambda$ are compared and analyzed. These efforts show that taking $\lambda=1.5$ is a more reasonable and reliable choice.
\end{abstract}

\section{Introduction}

Ferritic steels are widely used in lots of pivotal engineering structures such as reactor pressure vessels (RPVs), which need high requirements in anti-fracture safety [1]. However, there is ductile-tobrittle transition temperature characteristic of ferritic steels in essence [2]. At low temperatures, low stress embrittlement occurs [3]. One of the characteristics of embrittlement is randomness. So it is necessary to employ statistical fracture mechanics methods to study cleavage fracture in ferritic steels. Some methods had been proposed, such as the Maser Curve method and the local approach (LA). The Master Curve method was proposed by Wallin [4] and standardized by ASTM E1921 [5]. This method is used to determine the reference temperature $\mathrm{T}_{0}$ in ductile-to-brittle transition zone of ferrite steels. The disadvantage of the Master Curve mentioned in Qian et al. [6] is that in engineering applications, unlike in experiments, the crack front is constrained, which will lead to higher toughness and more conservative $\mathrm{T}_{0}$. This hinders the development of the Master Curve method and promotes the growth of micromechanical models. The local approach model (LAM) for cleavage fracture $[7,8]$ is a kind of micromechanical failure model, which is based on the weakest link theory [9]. One characteristic of cleavage fracture model used in LA is the adoption of Weibull stress to replace the conventional fracture mechanics parameters. Some basic features of the LA are shown below:

(1). Unlike fracture mechanics parameters, it is not constrained by plastic conditions.

(2). It naturally takes into account the influence of the sample size, and gives the failure probability under given conditions.

(3). The basic evaluation parameters of the LA model are obtained from tensile experiments and finite element (FE) calculations.

The Beremin model $[10,11]$ is one of the most widely used local approaches (LAs) to cleavage fracture, which obeys two-parameter Weibull distribution:

$$
\begin{aligned}
& P=1-\exp \left[-\left(\int_{V_{p l}} \sigma_{1}^{m} \cdot d V / V_{0}\right) / \sigma_{0}^{m}\right]=1-\exp \left[-\left(\sigma_{W} / \sigma_{0}\right)^{m}\right] \\
& \sigma_{W}=\left(\int_{V_{p l}} \sigma_{1}^{m} \cdot d V / V_{0}\right)^{1 / m}
\end{aligned}
$$

where $P$ denotes the cumulative failure probability, $V_{p l}$ denotes the integral plastic volume, $\sigma_{1}$ denotes the maximum tensile principal stress, $m$ is the Weibull modulus, $\sigma_{0}$ is the Weibull scale parameter. $V_{0}$ is the mean volume occupied by each microcrack in a solid, and $\sigma_{W}$ denotes the Weibull stress. In recent years, Beremin model has been

\footnotetext{
* Corresponding authors.

E-mail addresses: wiselee18@163.com (P. Li), qianguian@imech.ac.cn (G. Qian).
} 


\author{
Nomenclature \\ $d V \quad$ differential volume \\ E Young's modulus \\ FE finite element \\ FEA finite element analysis \\ $i \quad$ the serial number of the specimen \\ LA local approach \\ LAM local approach model \\ LR linear regression \\ $m \quad$ Weibull modulus \\ $N \quad$ the total number of the specimen \\ NLAM new local approach model \\ $P \quad$ cumulative probability of failure \\ $P_{i} \quad$ prescribed probability for the $i$ th specimen
}

$\begin{array}{ll}\text { RPVs } & \text { reactor pressure vessels } \\ V_{0} & \text { mean volume occupied by each microcrack in a solid } \\ V_{p l} & \text { plastic volume } \\ \lambda & \text { threshold coefficient of fracture process zone } \\ \nu & \text { Poisson's ratio } \\ \sigma_{0} & \text { Weibull scale parameter } \\ \sigma_{1} & \text { maximum tensile principal stress } \\ \sigma_{1,0} & \text { value of } \sigma_{1} \text { at initial yield of a differential volume element } \\ & d V \\ \sigma_{N} & \text { fracture stress } \\ \sigma_{V M} & \text { von-Mises stress } \\ \sigma_{W} & \text { Weibull stress } \\ \sigma_{W, \text { new }} & \text { newly defined Weibull stress } \\ \sigma_{y s} & \text { yield stress } \\ \sigma_{\infty} & \text { loading stress }\end{array}$

widely studied [12-18]. Before the Beremin model is put into any practical uses, $m$ and $\sigma_{0}$ need to be calibrated from a number of fracture experiments. The calibration procedure was proposed by Minami et al. [19] and ESIS [20]. The main problem is that the calibrated values of the Weibull parameters $m$ and $\sigma_{0}$ change with temperature and specimen dimension [21-24]. Long-term ambiguity of calibration seriously affects the improvement of Beremin model. To address this problem, a lot of works had been done. For instance, Ruggieri et al. [25] discussed the non-uniqueness in the calibrated Weibull parameters using a single set of specimen. They suggested using two sets of specimens with different constraints to determine a unique set of Weibull parameters and proposed a new parameters calibration scheme. Gao et al. [26], Petti and Dodds [27] and Hadid-Moud et al. [28] rewrote the formula of $P$ into a three-parameter Weibull distribution form, but these formulas lack strict theoretical derivation. Under the assumption that plastic yield is the prerequisite to cleavage fracture, Lei $[29,30]$ proposed a new probabilistic model:

$P=1-\exp \left\{-\int_{V p l}\left[\left(\sigma_{1}-\sigma_{1,0}\right)^{m} \cdot d V / V_{0}\right] / \sigma_{0}^{m}\right\}=1-\exp \left[-\left(\sigma_{W, \text { new }} / \sigma_{0}\right)^{m}\right]$

$\sigma_{W, \text { new }}=\left(\int_{V p l}\left(\sigma_{1}-\sigma_{1,0}\right)^{m} \cdot d V / V_{0}\right)^{1 / m}$

where $\sigma_{1,0}$ denotes the value of $\sigma_{1}$ at initial yield of a differential volume element $d V$, and $\sigma_{W, \text { new }}$ is the newly defined Weibull stress. Eq. (3) can be rewritten as follows:

$\operatorname{LnLn}[1 /(1-P)]=m \operatorname{Ln}\left(\sigma_{W, \text { new }}\right)-m \operatorname{Ln}\left(\sigma_{0}\right)$

from Eq. (5), we can see that there is a certain linear relationship between $\operatorname{LnLn}(1 /(1-P))$ and $\operatorname{Ln}\left(\sigma_{W}\right) . m$ can be obtained by the slope of Eq. (5) and $\sigma_{0}$ can be obtained by intercept. To calibrate Eq. (5), the well-developed linear regression (LR) method was allowed to obtain the accurate two Weibull parameters $m$ and $\sigma_{0}$.

As is mentioned in Lei [29], for ceramics and other brittle materials, with the increase of load, they can only undergo macroscopic elastic deformation before fracture. With the increase of external load, steels always undergo elastic deformation first and then whole or local plastic deformation. In addition, there is an essential difference between the cleavage fracture of metals and the fracture mode of brittle material. Before a brittle material is put into use, there exist some micro-defects, such as voids and grain boundary cracks. Once elastic stress triggers the unstable propagation of microdefects, fracture will occur. While in ferritic steels, there are few geometrical discontinuities in microstructure which preexist as metallurgical defects. The cleavage fracture process of ferritic steel involves two parts, one is the nucleation of microcracks and the other is the expansion of microcracks. Many microstructures can nucleate microcracks in a stressed volume, such as dislocation interaction, carbide cracking and so on. However, regardless of the mechanism by which microcracks nucleate, cleavage fracture is always caused by the plastic yield of ferrite grains [30]. In a word, it is a consensus that the prerequisite of cleavage fracture is plastic yield. Eq. (3) is desired to surmount the uncertain calibration of the two Weibull parameters because it strictly insists that the prerequisite of cleavage fracture is plastic yield. Compared with the Beremin model, improvements in the NLAM are as follows:

(1). Mathematically, under the assumption of uniform distribution of microcracks, the new model proposes a cumulative failure probability model. It is based on the hypothesis of the weakest link and it guarantees that the new model complies with the normality axiom of probability, a fundamental point of all probabilistic models. And the probabilistic model is self-consistent [31].

(2). Physically, based on Eq. (3), we can see that for any differential volume element $d V$, the probability of cumulative failure $P$ is calculated only by considering the stress increment $\left(\sigma_{1}-\sigma_{1,0}\right)$ after initial plastic yield. Therefore, the NLAM pertinently follows the fundamental physical assumption that the precondition for cleavage fracture is plastic yield [32].

Readers can refer to Lei $[29,30]$ to learn more about the characteristics of the NLAM. Here we are committed to finding out a simple method to calibrate the new model parameters. In Qian et al. [31], it was proven that the statistical model parameters for cleavage fracture in ferritic steels are temperature independent by using the NLAM. Although the new model with $\sigma_{1,0}$ overcomes the problems of the Beremin model, there is no mature and convenient method to estimate it at present. This work aims to find out a simplified method for parameters calibration of the NLAM by use of some published experimental fracture data-sets [33-35].

\section{Experimental data}

The cleavage fracture stress data of low alloy ferritic steel 20MnMoNi55 in Chakraborti et al. [35] are used, which were obtained from the tested circumferentially notched round tensile specimens shown in Fig. 1 under four subzero temperatures $(123 \mathrm{~K}, 173 \mathrm{~K}, 203 \mathrm{~K}$ and $223 \mathrm{~K}$ ). It should be noted that the circumferential notch in the Fig. 1 is semi-circular. The chemical composition and material properties of 20MnMoNi55 are given in Table 1 and 2, respectively.

The true stress-plastic strain curves of $20 \mathrm{MnMoNi} 55$ at four subzero temperatures reported in Manjunath et al. [34] are shown in Fig. 2. They act as the input data to FEA. In Chakraborti et al. [35], 30 groups of fracture stress data of $20 \mathrm{MnMoNi5} 5$ were tested at the above four different subzero temperatures. Ten circled points of fracture stress in Qian et al. [31] are used for FE calculation. The fracture stress $\sigma_{N}$ can be transformed into loading stress $\sigma_{\infty}$ via the formula $\sigma_{\infty}=\sigma_{N}\left(\phi_{2} / \phi_{1}\right)^{2}$, 


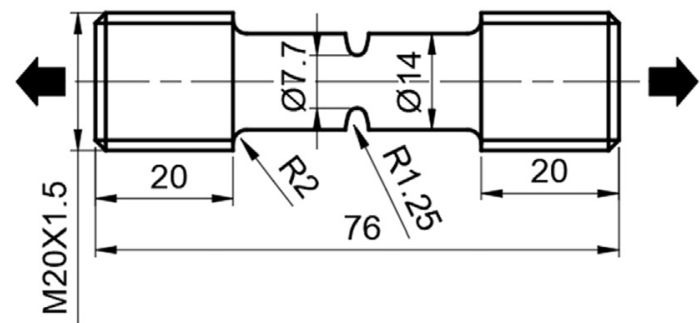

Fig. 1. Dimensions of circumferentially notched round tensile specimen (All dimensions are in $\mathrm{mm}$ ).

Table 1

Chemical composition of low alloy ferritic steel 20MnMoNi55 (wt pct) [35].

\begin{tabular}{lllllllllll}
\hline $\mathrm{C}$ & $\mathrm{Si}$ & $\mathrm{Mn}$ & $\mathrm{P}$ & $\mathrm{S}$ & $\mathrm{Ni}$ & $\mathrm{Gr}$ & $\mathrm{Mo}$ & $\mathrm{V}$ & $\mathrm{Al}$ & $\mathrm{Fe}$ \\
\hline 0.21 & 0.21 & 1.3 & 0.009 & 0.001 & 0.68 & 0.05 & 0.494 & 0.01 & 0.029 & balance
\end{tabular}

Table 2

Material properties of low alloy ferritic steel 20MnMoNi55 [33-35].

\begin{tabular}{ll}
\hline Young's modulus $E(\mathrm{GPa})$ & Poisson's ratio $v$ \\
\hline 200 & 0.3 \\
Temperature $(\mathrm{K})$ & Yield stress $\sigma_{y s}(\mathrm{MPa})$ \\
123 & 685 \\
173 & 600 \\
203 & 558 \\
223 & 537
\end{tabular}

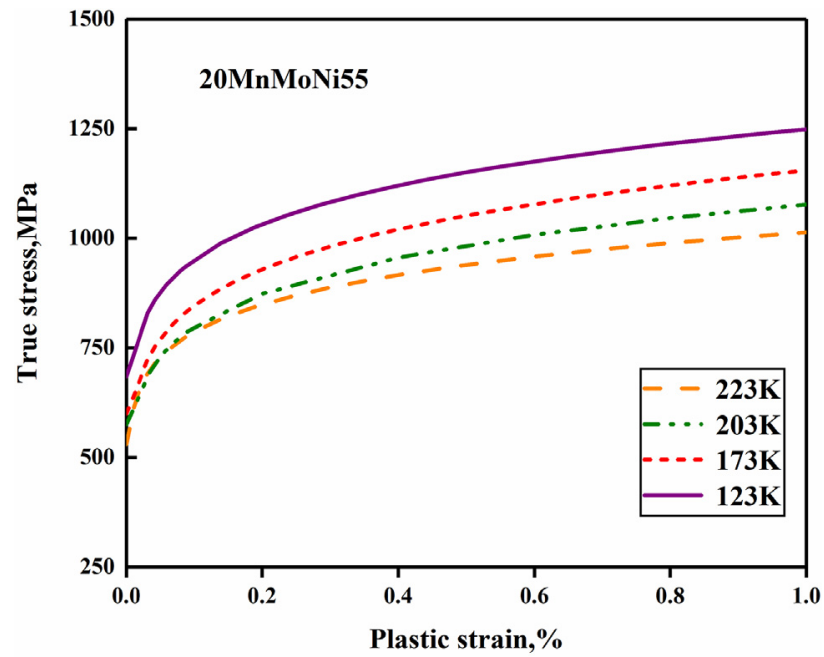

Fig. 2. True stress-plastic strain curves of $20 \mathrm{MnMoNi55}$ at four subzero temperatures $[33,35]$.

where the values of $\phi_{1}$ and $\phi_{2}$ are given in Fig. $1\left(\phi_{1}=14 \mathrm{~mm}\right.$, and $\phi_{2}=7.7 \mathrm{~mm}$ ).

\section{FE simulations and parameters calibration}

\subsection{FE simulations}

In this study, the 3D elastoplastic stress analysis of the specimen is carried out by using FEA software ABAQUS. ABAQUS version 6.14 is used for all simulations and the 20-node brick elements are used for analysis. Considering the uniform symmetry of the structure and the loading load, only $1 / 8$ of the sample is modeled and symmetric constraints are applied on the symmetrical plane of the specimen to reduce FE calculation time. The number of elements and nodes are 146,000 and 341707 , respectively. The minimum element size is $0.001 \mathrm{~mm}^{3}$. It should be noted that at the edge of the notch the grid should be more denser. As mentioned in Qian et al. [32], the FEA can be divided into two steps. The first step is using the elastic-perfectly plastic material defined by Poisson's ratio $\nu$, the Young's modulus $E$ and yield stress $\sigma_{y s}$ to obtain the initial yield stress $\sigma_{1,0}$ mentioned in Eq. (3). The second step is the elastoplastic analysis using the true stress-strain curves of the material shown in Fig. 2. The true stress- strain curves are used as input to FEA. When defining properties of material in ABAQUS, we choose elastic and plastic as the material behaviors. Elastic material is defined by the Young's modulus $E$ and Poisson's ratio $v$, the plastic part is defined by the true stress-strain data. Partial results of the maximum principal stress of FEA are presented in the following Fig. 3. The distributions of $\sigma_{1,0}$ in the specimen at two subzero temperatures are shown in Fig. 4.

\subsection{Threshold coefficient of fracture process $\lambda$}

It should be pointed out that the Beremin research group did not clearly define the fracture process zone when they established the LAM of cleavage fracture. When the model was derived, it was considered that the microcrack in the plastic zone causes cleavage fracture, so the element contributing to Weibull stress should be the element in the plastic zone. That is, the maximum principal stress $\sigma_{1}$ satisfies the formula: $\sigma_{1} \geqslant \sigma_{y s}$. Ruggieri and Dodds [36] discussed the fracture zone of the crack body in detail, and they defined that the fracture process zone should be the zone satisfying the following condition: $\sigma_{1} \geqslant \lambda \sigma_{y s}$, where $\lambda$ is the threshold coefficient of fracture process and $\lambda \geqslant 1$. In Ruggieri and Dodd [36], $\lambda$ was taken as 2. To investigate the effect of $\lambda$ on the calculation results of Weibull stress, Hui [37] took 1 and 2 as the values of $\lambda$ in the cracked specimen by using traditional LAM, and found that $\lambda$ has little influence on the calculation results. Qian et al. [31] proposed that the fracture process zone should be replaced by the plastic zone defined by the von-Mises yield criterion. When determining the integral volume, they chose the volume under the criterion $\sigma_{V M} \geqslant 1.5 \sigma_{y s}$ rather than the more accurate volume defined by the strict von-Mises yield criterion $\sigma_{V M} \geqslant \sigma_{y s}$, where $\sigma_{V M}$ denotes the von-Mises stress. However, adopting which appropriate value of $\lambda$ in the NLAM remains a problem.

The main purpose of this work is to find out a suitable value of $\lambda$. It can not only reduce the computational time but also guarantee relatively accurate Weibull parameters. In this study, the integral volume $V_{p l}$ is defined by the criterion $\sigma_{V M} \geqslant \lambda \sigma_{y s}, \lambda$ is taken as $1,1.25,1.5$, and 1.75 to calculate the plastic volume, respectively. It should be mentioned that we don't take $\lambda=2$ as mentioned in Hui [37] because the stress concentration factor in the plastic zone of blunt notched specimen under consideration in this work is usually much smaller than that of the cracked specimen studied by Hui [37].

\subsection{Calibration method}

For the true constitutive relationship of the material, the number of elements, the volume of the elements, the maximum principal stress of each element and the von-Mises stress can be obtained from the results of the above FE simulations under ten different loading stresses at $123 \mathrm{~K}$ and $223 \mathrm{~K}$. For elastic-perfectly plastic material, the loading stresses of $380 \mathrm{MPa}$ and $280 \mathrm{MPa}$ are applied on the specimens to calculate $\sigma_{1,0}$ at $123 \mathrm{~K}$ and $223 \mathrm{~K}$, respectively. Since the values of $\sigma_{1,0}, \sigma_{1}$ and $d V$ are known, to calculate $\sigma_{W, \text { new }}$ defined in Eq. (4), the mean volume $V_{0}$ occupied by each microcrack in a solid and Weibull parameter $m$ should be constants. This work takes the starting value of the $m$ as 10 and $V_{0}$ is taken as $0.001 \mathrm{~mm}^{3}$.

After calculating $\sigma_{W, \text { new }}$ in Eq. (4), the two Weibull parameters ( $m$ and $\sigma_{0}$ ) will be determined by the linear method in Eq. (5) by using the calibration procedure proposed by Qian et al. [38] as illustrated in Fig. 5. 


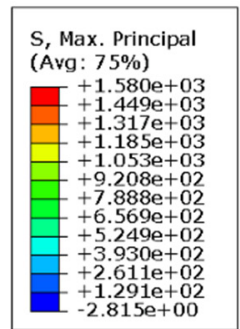

(a)

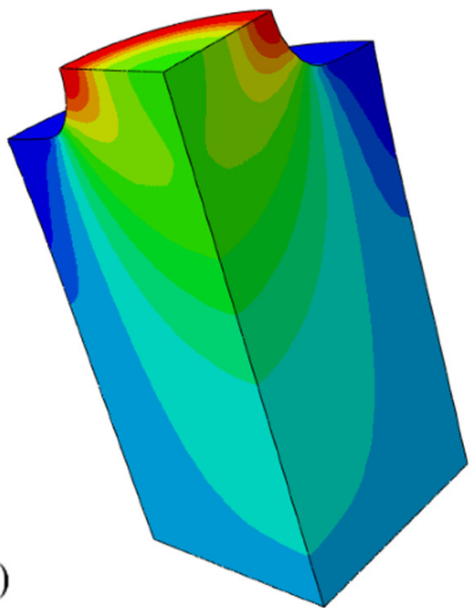

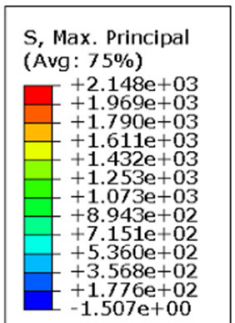

(b)

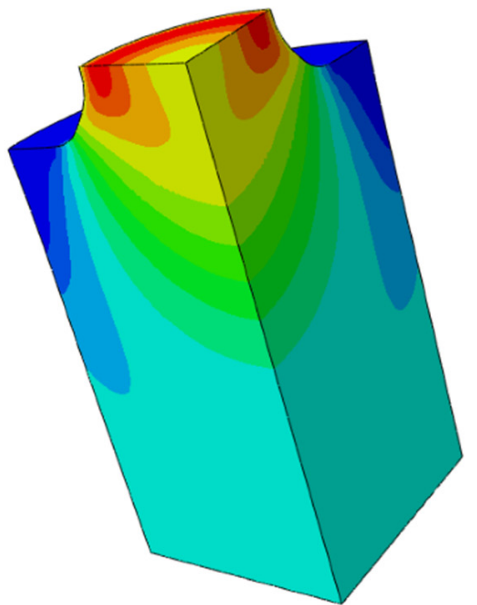

Fig. 3. Maximum tensile principal stress $\sigma_{1}$ distributions in the specimen at $123 \mathrm{~K}$ : (a) $\sigma_{\infty}=386 \mathrm{MPa}$; (b) $\sigma_{\infty}=556 \mathrm{MPa}$; true elastoplastic hardening model.
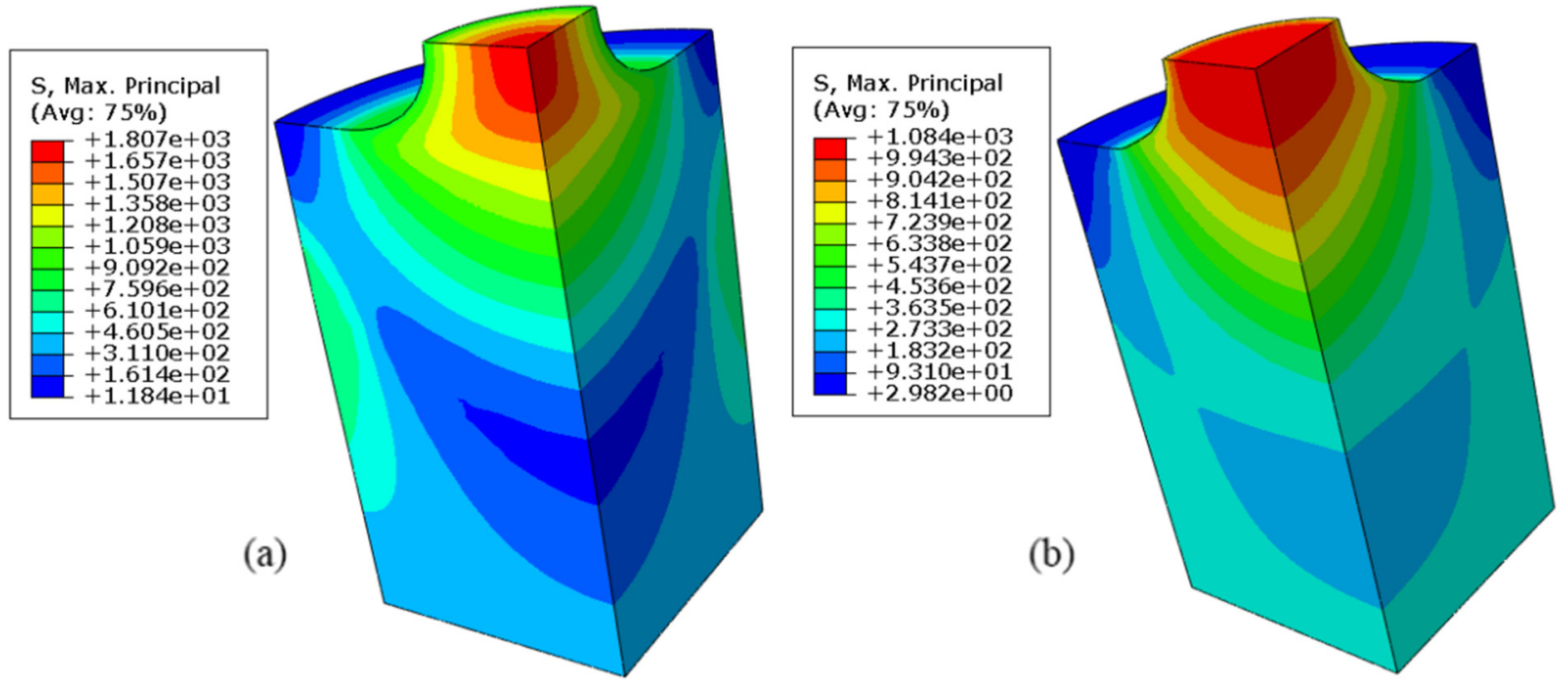

Fig. 4. The distributions of $\sigma_{1,0}$ in the specimen based on the elastic-perfectly plastic constitutive model: (a) at $123 \mathrm{~K}$; (b) at $223 \mathrm{~K}$.

Elastic-perfectly plastic FEA to obtain $\sigma_{1,0, \mathrm{i}}$ for each volume element $\Delta \mathrm{V}_{\mathrm{i}}$

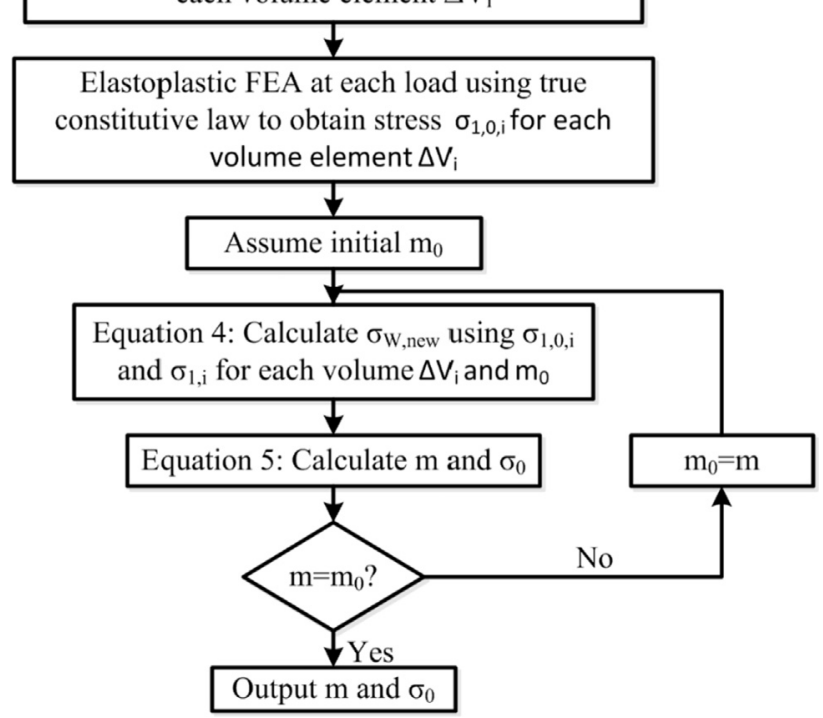

Fig. 5. Flow chart of the calibration procedure for NLAM.

\section{Results and discussion}

The results of logarithmic LR in Eq. (5) by using the calibration procedure shown in Fig. 5 under four different values of $\lambda$ at $123 \mathrm{~K}$ are presented in Fig. 6, and the results at $223 \mathrm{~K}$ are illustrated in Fig. 7. In order to solve the problem that $P$ is unknown in Eq. (5), Minami et al. [19] proposed a prescribed probability estimator, which is $P_{i}=(i-0.5) / N$, where $i$ is the number of the specimen, $i=1,2 . . . N(N=10)$. The slope of a straight line in Fig. 6 and Fig. 7 is the calibration value of $m$. Then, the calibrated results are summarized in Table 3. From Table 3, we can see clearly that at $123 \mathrm{~K}$ and $223 \mathrm{~K}$, different values of $\lambda$ correspond to different $m$ and $\sigma_{0}$.

Now we manage to provide the method to determine the appropriate value of $\lambda$. For the convenience of statement, we might as well take $\lambda_{A}=1.0$ and $\lambda_{B}=1.5$, and the corresponding calibrated values of $m$ and $\sigma_{0}$ are $m_{A}$ and $\sigma_{0, A}, m_{B}$ and $\sigma_{0, B}$ respectively. Remembering the criterion $\sigma_{V M} \geqslant \lambda \sigma_{y s}$, we have $\sigma_{V M, A} \geqslant \lambda_{A} \sigma_{y s}$, and $\sigma_{V M, B} \geqslant \lambda_{B} \sigma_{y s}$. Prior to comparing $\lambda_{A}$ and $\lambda_{B}$, we shall highlight the following two requirements:

(1). The value of $m$ varies within a small range when $\lambda$ changes from $\lambda_{A}=1.0$ to $\lambda_{B}=1.5$.

(2). The comprehensive effect caused by $m$ and $\sigma_{0}$ say $\left(\int_{V p l}\left(\frac{\sigma_{1}-\sigma_{1,0}}{\sigma_{0}}\right)^{m} \frac{d V}{V_{0}}\right)$ varies fairly small as $\lambda$ increases. In other words, 


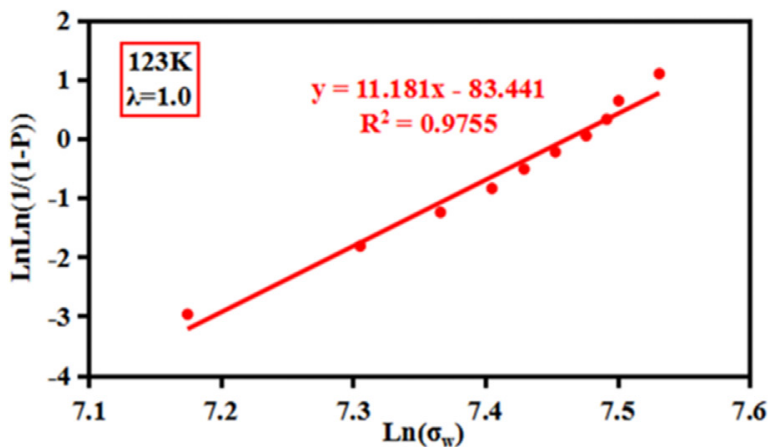

(a)

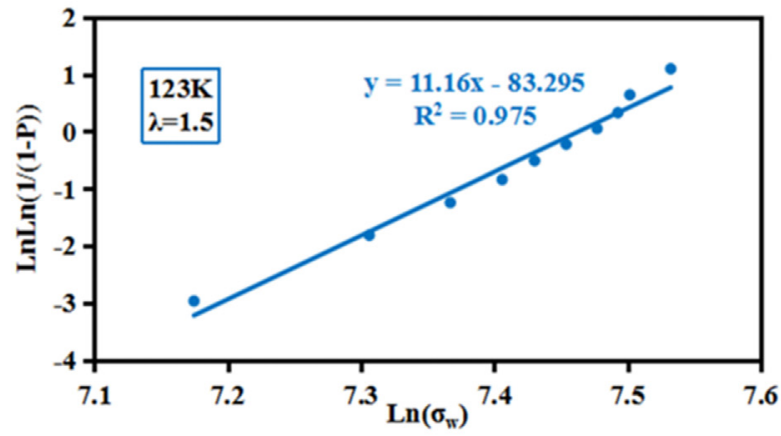

(c)

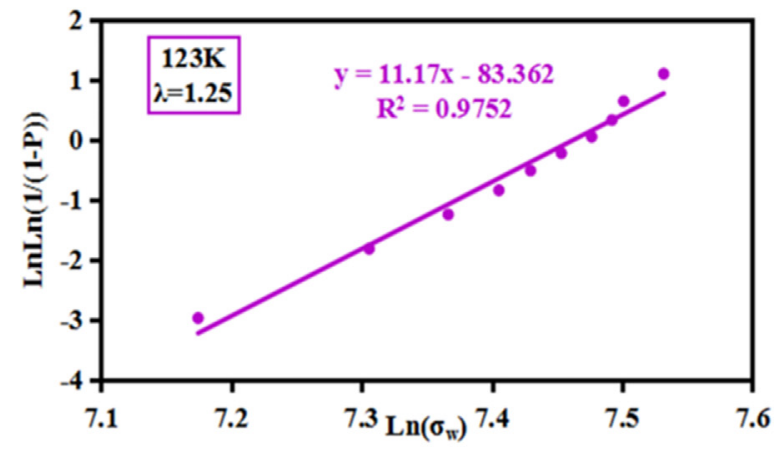

(b)

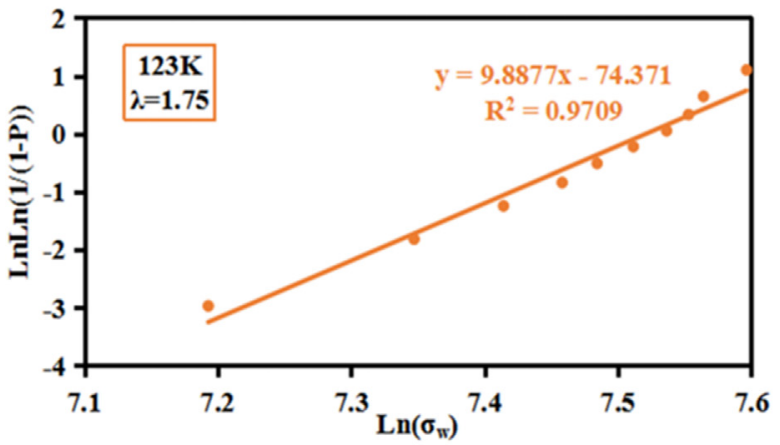

(d)

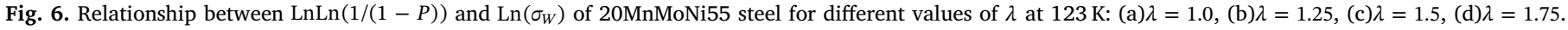

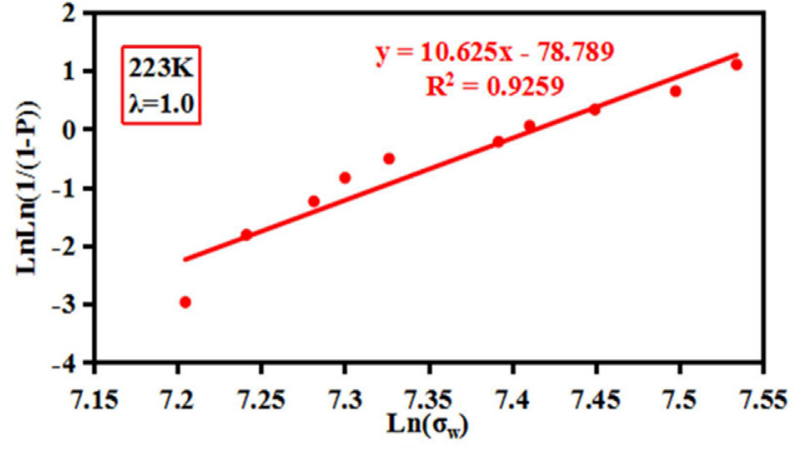

(a)

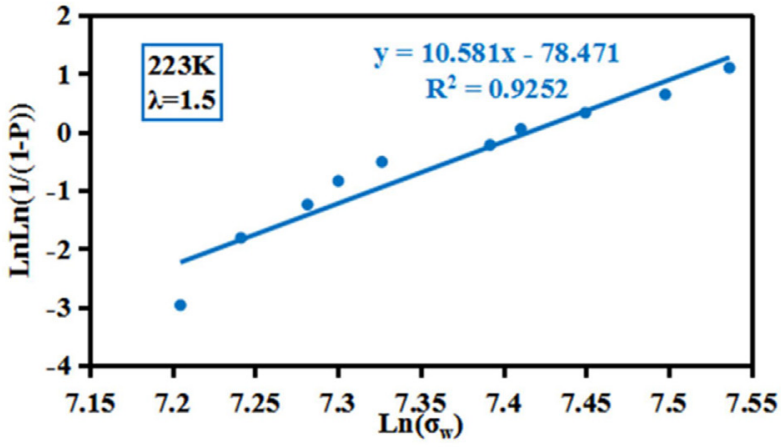

(c)

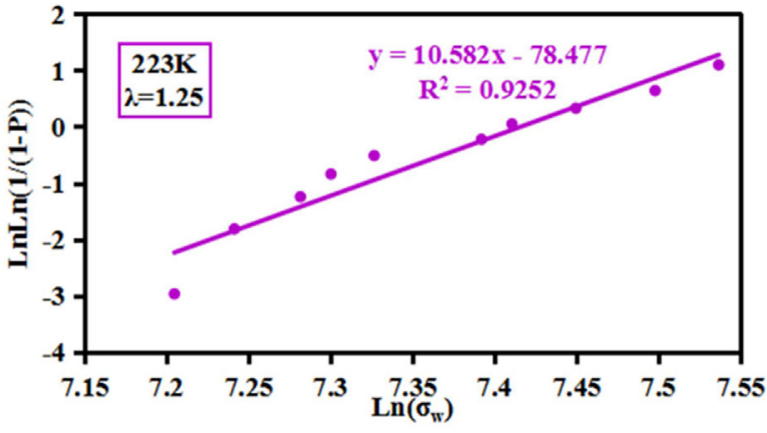

(b)

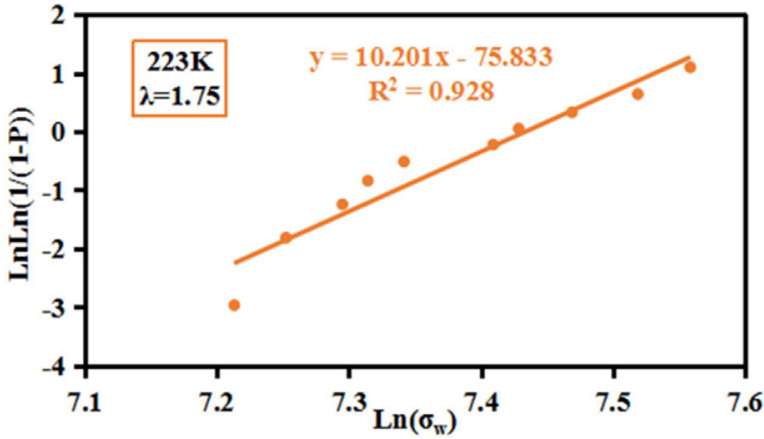

(d)

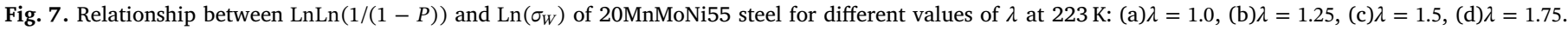

$\int_{V p l}\left(\frac{\sigma_{1}-\sigma_{1,0, A}}{\sigma_{0, A}}\right)^{m_{A}} \frac{d V}{V_{0}} \approx \int_{V p l}\left(\frac{\sigma_{1}-\sigma_{1,0, B}}{\sigma_{0, B}}\right)^{m_{B}} \frac{d V}{V_{0}}$

It can be clearly seen from Table 3 that the values of $m$ and $\sigma_{0}$ change slightly when $\lambda$ increases from 1.0 to 1.5 . At $123 \mathrm{~K}$, the variation of $m$ is $0.19 \%$, and that of $\sigma_{0}$ is only $0.1 \%$. In the case of $223 \mathrm{~K}$, as $\lambda$ ranges from 1.0 to 1.5 , there is $0.41 \%$ change in $m$ and $0.08 \%$ increase in $\sigma_{0}$. However, it is not the case when $\lambda$ grows from 1.0 to 1.75 . At $123 \mathrm{~K}$, we see that $m$ decreases by $11.57 \%$ and $\sigma_{0}$ increases by $6.06 \%$. Besides, at $223 \mathrm{~K}$, there is a $3.99 \%$ reduction in the value of $m$ and a $1.86 \%$ increase in the value of $\sigma_{0}$. Compared to the case of $\lambda=1.5$, we 
Table 3

Calibration results of $m$ and $\sigma_{0}$ for different values of $\lambda$.

\begin{tabular}{|c|c|c|c|c|c|c|c|c|}
\hline \multirow[b]{2}{*}{$\mathrm{T}(\mathrm{K})$} & \multicolumn{2}{|l|}{$\lambda=1.0$} & \multicolumn{2}{|l|}{$\lambda=1.25$} & \multicolumn{2}{|l|}{$\lambda=1.5$} & \multicolumn{2}{|c|}{$\lambda=1.75$} \\
\hline & $m$ & $\sigma_{0}(\mathrm{MPa})$ & $m$ & $\sigma_{0}(\mathrm{MPa})$ & $m$ & $\sigma_{0}(\mathrm{MPa})$ & $m$ & $\sigma_{0}(\mathrm{MPa})$ \\
\hline $123 \mathrm{~K}$ & 11.181 & 1741.93 & 11.17 & 1742.41 & 11.16 & 1743.60 & 9.8877 & 1847.46 \\
\hline $223 \mathrm{~K}$ & 10.625 & 1661.43 & 10.582 & 1662.51 & 10.581 & 1662.73 & 10.201 & 1692.36 \\
\hline
\end{tabular}

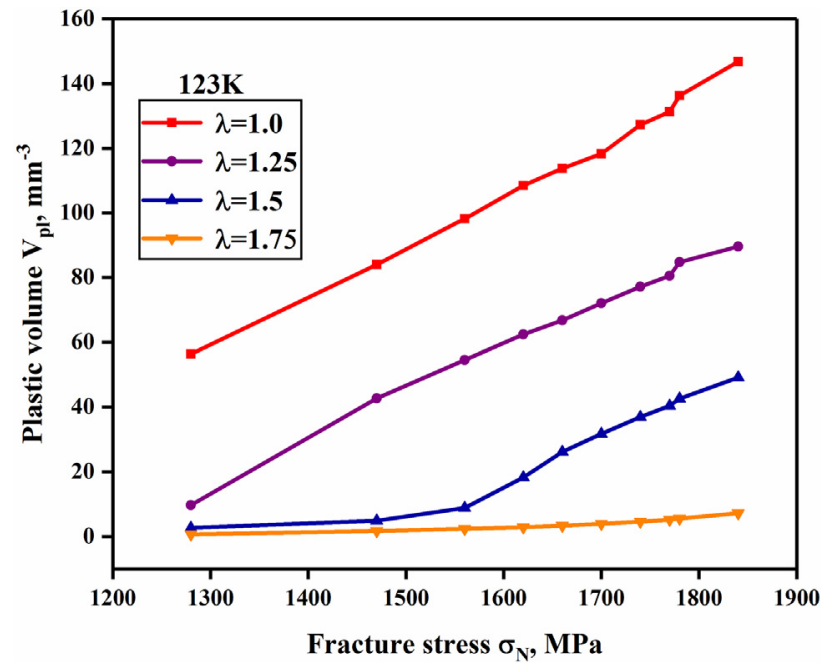

Fig. 8. Variation of $V_{p l}$ with $\sigma_{N}$ at $123 \mathrm{~K}$ for different values of $\lambda$.

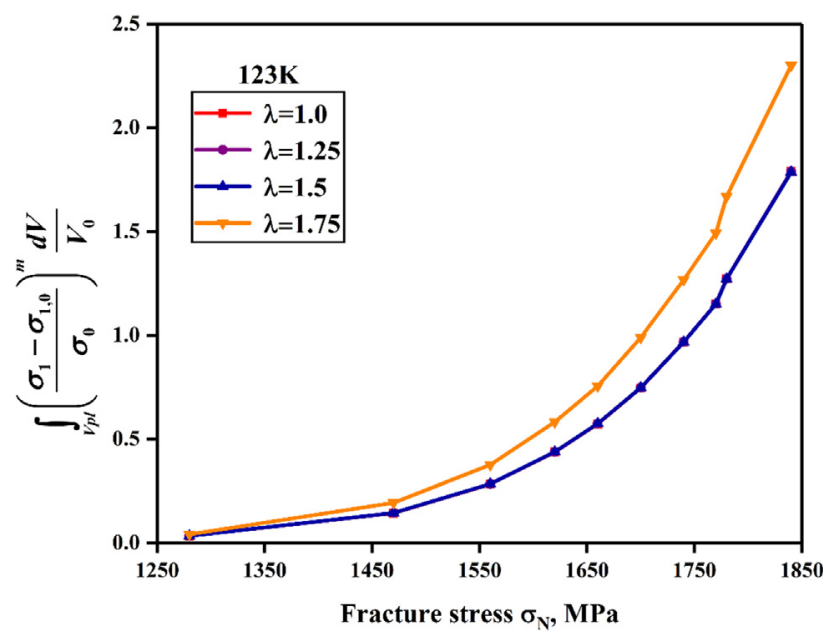

Fig. 9. Variation of the comprehensive effect with $\sigma_{N}$ at $123 \mathrm{~K}$ for different values of $\lambda$.

can see that the accuracy of the calibration is reduced for $\lambda=1.75$. Concerning this point, we give the following explanation. As for the case of $\lambda=1.75$, we adopt the criterion $\sigma_{V M} \geqslant 1.75 \sigma_{y s}$ by default. Probably, that implies there are too many FE volumes ignored when adopting the above criterion to calculate the plastic volume $V_{p l}$. In other words, considering that the criterion $\sigma_{V M} \geqslant 1.75 \sigma_{y s}$ is much more demanding than $\sigma_{V M} \geqslant 1.0 \sigma_{y s}$, the integral volume $V_{p l}$ in Eq. (4) for $\lambda=1.75$ is much smaller than that for $\lambda=1.0$. This will result in computational distortion. Fig. 8 displays the variation of plastic volume $V_{p l}$ with the fracture stress under four different values of $\lambda$ at $123 \mathrm{~K}$. It is clearly seen from the curves in Fig. 8 that $V_{p l}$ is severely underestimated in the situation of $\lambda=1.75$.

Next, we turn to the issue of the comprehensive effect induced by $m$ and $\sigma_{0}$. The variation of the comprehensive effect with $\sigma_{N}$ at two subzero temperatures $(123 \mathrm{~K}$ and $223 \mathrm{~K})$ for different values of $\lambda$ are

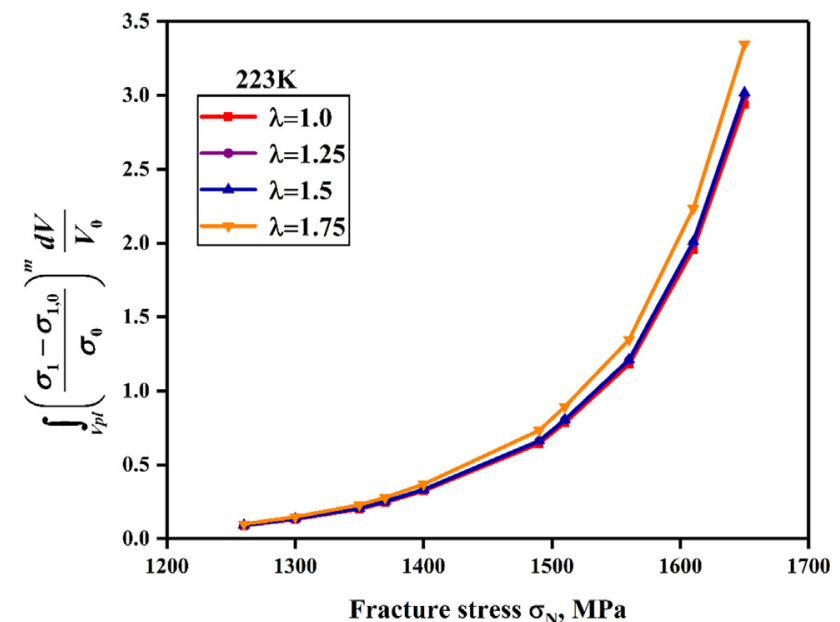

Fig. 10. Variation of the comprehensive effect with $\sigma_{N}$ at $223 \mathrm{~K}$ for different values of $\lambda$.

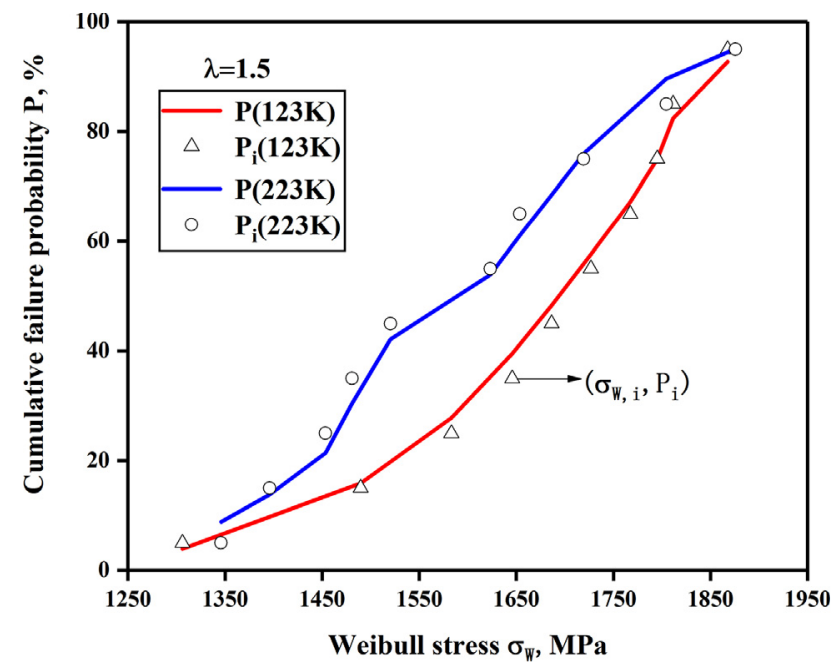

Fig. 11. Comparison between the experimental results with the calibrated results of $P$.

plotted in Fig. 9 and 10.

Observing the curves in the above mentioned two figures, we find that the three curves corresponding to the three cases of $\lambda=1.0$, $\lambda=1.25$ and $\lambda=1.5$ almost coincide with one another. This indicates there is no evident change in the combined effect when the value of $\lambda$ ranges from 1 to 1.5. But, when it comes to the case of $\lambda=1.75$, there is an obvious deviation from the other three curves, especially when it is $123 \mathrm{~K}$.

In order to further distinguish the more delicate difference in the comprehensive effect under different values of $\lambda$, we provide the detailed results as follows. With $\lambda$ increasing from 1.0 to 1.25 , the maximum change value of $\int_{V p l}\left(\frac{\sigma_{1}-\sigma_{1,0}}{\sigma_{0}}\right)^{m} \frac{d V}{V_{0}}$ is $0.43 \%$ at $123 \mathrm{~K}$ and $2.88 \%$ at $223 \mathrm{~K}$. As $\lambda$ increases from 1.0 to 1.5 , the maximum change of $\int_{V p l}\left(\frac{\sigma_{1}-\sigma_{1,0}}{\sigma_{0}}\right)^{m} \frac{d V}{V_{0}}$ is $0.44 \%$ at $123 \mathrm{~K}$ and $3.49 \%$ at $223 \mathrm{~K}$. That is, the 
relative error is within $4 \%$ for $\lambda=1.25$ and $\lambda=1.5$. However, for the case of $\lambda$ increasing from 1.0 to 1.75, the maximum change value of $\int_{V p l}\left(\frac{\sigma_{1}-\sigma_{1,0}}{\sigma_{0}}\right)^{m} \frac{d V}{V_{0}}$ becomes $33.17 \%$ at $123 \mathrm{~K}$ and $14.15 \%$ at $223 \mathrm{~K}$. Obviously, this is much higher than the above $4 \%$ and seems to be unreasonable.

In summary, it comes to the conclusion that when $\lambda=1.25$ and $\lambda=1.5$, the calibrated results of $m$ and $\sigma_{0}$ are both close to the results with $\lambda=1.0$, and the deviation of the comprehensive effect under the corresponding $\lambda$ is small (with error band of $4 \%$ ). However, it can be clearly seen from Fig. 8 that the plastic volume under the criterion $\sigma_{V M} \geqslant 1.5 \sigma_{y s}$ is much smaller than that under $\sigma_{V M} \geqslant 1.25 \sigma_{y s}$. This means that when $\lambda=1.5$, the computational workload and the computation time will be greatly shortened. Based on the above results, we can see that taking $\lambda=1.5$ is a good way when calculating the plastic volume. Once the values of $m, \sigma_{0}$ and $\sigma_{W}$ are determined by the supposed value of $\lambda$, we can substitute them into the NLAM to predict the failure probability.

Fig. 11 compares the experimental results with the calibrated results of the cumulative failure probability. The empty dots are experimental results of the cumulative failure probability, the cumulative failure probability here doesn't mean the failure probability obtained from the experiment but calculated by the formula $P_{i}=(i-0.5) / N$, where $P_{i}$ is the rank probability. $\sigma_{W, i}$ is the Weibull stress corresponding to the $i$ th fracture load value of $N$ fracture load measurements arranged in an ascending order. The solid lines are based on Eq. (3), with the calibrated values of $m$ and $\sigma_{0}$ when taking $\lambda=1.5$ as input. The red line represents the result of the input of $m=11.16, \sigma_{0}=1743.60 \mathrm{MPa}$ at $123 \mathrm{~K}$, while the blue one denotes the result of the input of $m=10.581$, $\sigma_{0}=1662.73 \mathrm{MPa}$ at $223 \mathrm{~K}$. In both cases, we can see that the two sets of results agree well, which indicates that taking $\lambda=1.5$ is a reasonable option.

\section{Conclusions}

This study uses the tensile test data of axisymmetric notched specimen 20MnMoNi55 steel under two subzero temperatures for FEA to obtain the stress distributions, and four groups of Weibull parameters under different $\lambda$ are obtained. After investigating and comparing the above four groups of calibration results, the major conclusions can be drawn as follows:

(1). When the threshold coefficient $\lambda$ increases from 1.0 to 1.5 , the calibrated $m$ and $\sigma_{0}$ of the NLAM show little change at both $123 \mathrm{~K}$ and $223 \mathrm{~K}$. The comprehensive effect caused by $m$ and $\sigma_{0}$ hardly changes with $\lambda$ ranging from 1.0 to 1.5 under different temperatures.

(2). The integral volume is greatly reduced by adopting $\lambda=1.5$. The calibrated result of the cumulative failure probability is close to that of the experimental result when taking $\lambda=1.5$.

(3). A simple approach to calibrate the NLAM has been proposed. That is, it is appropriate to take $\lambda=1.5$ as the threshold coefficient of fracture process zone when calculating the volume of the plastic deformation zone. This approach is time-saving and is of benefit to obtain relatively accurate Weibull parameters.

The present work is to find out a simplified calibration method for the new LA model which proved to be able to overcome the ambiguity calibration of Weibull parameters. As a preliminary study, the approach presented in this work is only based on two sets of experimental data available in the literature. So more pertinent experimental data are suggested to be supplemented so as to further validate the robustness of the presented approach. In our future work, we will use more experimental data to further study the new model and further verify the approach proposed in this work.

\section{Acknowledgement}

This work was funded by the National Natural Science Foundation of China (No.11872364).

\section{References}

[1] G. Qian, M. Niffenegger, M. Sharabi, N. Lafferty, Effect of non-uniform reactor cooling on fracture and constraint of a reactor pressure vessel, Fatigue Fract. Eng. Mater. Struct. 41 (2018) 1559-1575.

[2] Z. Zhang, X. Qian, Effect of experimental sample size on local Weibull assessment of cleavage fracture for steel, Fatigue Fract. Eng. Mater. Struct. 40 (7) (2017) 1128-1142.

[3] M. Moattari, I. Sattari-Far, I. Persechino, N. Bonora, Prediction of fracture toughness in ductile-to-brittle transition region using combined CDM and Beremin models, Mater. Sci. Eng., A 657 (2016) 161-172.

[4] K. Wallin, Master curve analysis of the "Euro" fracture toughness dataset, Eng Fract. Mech. 69 (4) (2002) 451-481.

[5] ASTM-E1921-02, Standard test method for determination of reference temperature, T0, for ferritic steels in the transition range. 2002

[6] G. Qian, V.F. González-Albuixech, M. Niffenegger, Calibration of Beremin model with the master curve, Eng. Fract. Mech. 136 (2015) 15-25.

[7] G. Qian, Y. Cao, M. Niffenegger, Yu.h.J. Chao, W. Wu, Comparison of constraint analyses with global and local approaches under uniaxial and biaxial loadings, Eur. J. Mech. A-Solid. 69 (135-146) (2018) 4.

[8] G. Qian, J. Zhai, Z. Yu, W. Lei, W. Wu, Non-proportional size scaling of strength of concrete in uniaxial and biaxial loading conditions, Fatigue Fract. Eng. Mater. Struct. 41 (2018) 1733-1745.

[9] K. Hojo, N. Ogawa, T. Hirota, K. Yoshimoto, Y. Nagoshi, S. Kawabata, Application of coupled damage and Beremin model to ductile-brittle transition temperature region considering constraint effect, Procedia Struct Integrity 2 (2016) 1643-1651.

[10] F.M. Beremin, A. Pineau, F. Mudry, et al., A local criterion for cleavage fracture of a nuclear pressure vessel steel, Metall. Trans A. 14 (11) (1983) 2277-2287.

[11] A. Pineau, Development of the local approach to fracture over the past 25 years: theory and applications, Int. J. Fract. 138 (1-4) (2006) 139-166.

[12] A. Andrieu, A. Pineau, J. Besson, D. Ryckelynck, O. Bouaziz, Beremin model: Methodology and application to the prediction of the Euro toughness data set, Eng. Fract. Mech. 95 (2012) 102-117.

[13] R. Beleznai, S. Szavai, Application of the Beremin model for cruciform specimen to determine the fracture probability in case of WPS, Strength Mater. 45 (4) (2013) 489-494.

[14] R.J. Smith, A.H. Sherry, A.C. Bannister, A.J. Horn, Prediction of SENB fracture toughness from Charpy data using the Beremin model in the lower transition region, in: Proceedings of the ASME 2013 Pressure Vessels and Piping Conference, Volume 6B: Materials and Fabrication. Paris, France (Paper No. PVP2013-97517, pp. V06BT06A029; http://doi.org/10 1115/PVP2013-97517), 2013. http:// proceedings.asmedigitalcollection. asme.org/proceeding.aspx? articleID =1815755.

[15] F. Minami, Implementation of Weibull stress to fracture assessment standards, Proc. Mater. Sci. 3 (2014) 337-343.

[16] S. Yoshizu, H. Nakai, K. Shibanuma, H. Yoshinari, S. Aihara, Probabilistic fracture mechanics analysis on the scatter of critical CTOD, Proc. Mater. Sci. 3 (2014) $1447-1452$.

[17] H. Moshayedi, I. Sattari-Far, The effect of welding residual stresses on brittle fracture in an internal surface cracked pipe, Int. J. Pressure Vessels Piping 126-127 (2015) 29-36.

[18] C. Ruggieri, Jr R H D. An engineering methodology for constraint corrections of elastic-plastic fracture toughness-Part I: A review on probabilistic models and exploration of plastic strain effects. Eng. Fract. Mech. 2015;134:368-390.

[19] F. Minami, A. Brückner-Foit, D. Munz, B. Trolldenier, Estimation procedure for the Weibull parameters used in the local approach, Int. J. Fract. 54 (3) (1992) 197-210.

[20] European Structural Integrity Society (ESIS), Procedure to measure and calculate materials parameters for the local approach to fracture using notch tensile specimens, European Structural Integrity Publication. 1998, ESIS P6-98: http://www. lavoisier.eu/books/other/esis-procedure-p6-98-procedure-to-measure-etcalculatematerial-parameters-for-the-local-approach-to-fracture-usingnotchedtensile-specimens/description_2272290.

[21] P.P. Milella, N. Bonora, On the dependence of the Weibull exponent on geometry and loading conditions and its implications on the fracture toughness probability curve using a local approach criterion, Int. J. Fract. 104 (1) (2000) 71-87.

[22] B. Wasiluk, J.P. Petti, R.H.D. Jr, Temperature dependence of Weibull stress parameters: studies using the Euro-material, Eng Fract Mech. 73 (8) (2006) 1046-1069.

[23] J.P. Mathieu, K. Inal, S. Berveiller, O. Diard, A micromechanical interpretation of the temperature dependence of Beremin model parameters for french RPV steel, J. Nucl. Mater. 406 (1) (2010) 97-112.

[24] Y. Cao, H. Hui, G. Wang, F.-Z. Xuan, Inferring the temperature dependence of Beremin cleavage model parameters from the Master Curve, Nucl. Eng. Des. 241 (1) (2011) 39-45.

[25] C. Ruggieri, X. Gao, R.H. Dodds, Transferability of elastic-plastic fracture toughness using the Weibull stress approach: significance of parameter calibration, Eng. Fract. Mech. 67 (2) (2000) 101-117.

[26] X. Gao, G. Zhang, T.S. Srivatsan, Prediction of cleavage fracture in ferritic steels: a modified Weibull stress model, Mater. Sci. Eng., A 394 (1) (2005) 210-219.

[27] J.P. Petti, R.H. Dodds, Calibration of the Weibull stress scale parameter, using the Master Curve, Eng. Fract. Mech. 72 (1) (2005) 91-120. 
[28] S. Hadidi-Moud, A. Mirzaee-Sisan, C.E. Truman, D.J. Smith, A local approach to cleavage fracture in ferritic steels following warm pre-stressing, Fatigue Fract. Eng. Mater. Struct. 27 (10) (2010) 931-942.

[29] W.-S. Lei, A cumulative failure probability model for cleavage fracture in ferritic steels, Mech. Mater. 93 (2016) 184-198.

[30] W.-S. Lei, A framework for statistical modelling of plastic yield initiated cleavage fracture of structural steels, Phil. Mag. 96 (35) (2016) 3586-3631.

[31] G. Qian, W.-S. Lei, M. Niffenegger, V.F. Gonzálezalbuixech, On the temperature independence of statistical model parameters for cleavage fracture in ferritic steels, Phil. Mag. 98 (2018) 959-1004.

[32] G. Qian, W.-S. Lei, M. Niffenegger, Calibration of a new local approach to cleavage fracture of ferritic steels, Mater. Sci. Eng., A 694 (2017) 10-12.

[33] B.K. Dutta, S. Guin, M.K. Sahu, M.K. Samal, Temperature dependency of Beremin's parameters for 20MnMoNi55 material. Paper\#G01/5, in: Transactions of SMiRT 19 Conference, International Association for Structural Mechanics in Reactor Technology, Toronto, Canada, 2007. Available at https://www.iasmirt.org/ transactions/19/G01_5.pdf. 2007;3: 255-260.
[34] B.S. Manjunath, P.V. Durgaprasad, B.K. Dutta, S.P. Prakash, Determination of Beremin's parameters for 20MnMoNi55 ferritic steel. Paper\# 129, in Transactions of SMiRT 21 Conference, International Association for Structural Mechanics in Reactor Technology, New Delhi, India, 2011. Available at https://www.iasmirt. org/transactions/21/p129.pdf.

[35] P.C. Chakraborti, A. Kundu, B.K. Dutta, Weibull analysis of low temperature fracture stress data of 20MnMoNi55 and SA333 (Grade 6) steels, Mater. Sci. Eng., A 594 (1) (2014) 89-97.

[36] C. Ruggieri, R.H. Dodds, A transferability model for brittle fracture including constraint and ductile tearing effects: a probabilistic approach, Int. J. Fract. 79 (4) (1996) 309-340.

[37] H. Hui, Study on key technology improvement and application of local approach for cleavage fracture. Ph.D. dissertation. East China University of Science and Technology. 2002. (In Chinese).

[38] G. Qian, W.-S. Lei, L. Peng, Z. Yu, M. Niffenegger, Statistical assessment of notch toughness against cleavage fracture of ferritic steels, Fatigue Fract. Eng. Mater. Struct. 41 (7) (2017) 1-12. 